\title{
Cell cycle regulating proteins p21 and p27 in prognosis of oral squamous cell carcinomas
}

\author{
THOMAS FILLIES $^{1}$, MIKE WOLTERING ${ }^{1}$, BURKHARD BRANDT ${ }^{3}$, JEAN-PAUL VAN DIEST $^{4}$, \\ RICHARD WERKMEISTER ${ }^{5}$, ULRICH JOOS ${ }^{1}$ and HORST BUERGER ${ }^{2}$
}

\begin{abstract}
${ }^{1}$ Department of Cranio-Maxillofacial Surgery, ${ }^{2}$ Institute of Pathology, University of Münster, Münster; ${ }^{3}$ Institute of Tumour Biology, University of Hamburg, Hamburg, Germany; ${ }^{4}$ Department of Pathology, University Medical Center, Utrecht, The Netherlands; ${ }^{5}$ Department of Cranio-Maxillofacial Surgery, Central Army Hospital Koblenz, Germany
\end{abstract}

Received July 27, 2006; Accepted October 16, 2006

\begin{abstract}
Cyclin-dependent kinases (CDKs) and cyclindependent kinase inhibitors (CDKIs), such as p21 and p27, exert a direct control on the cell cycle.p21 and p27 are negative regulators of cyclin-dependent kinases and in this function they are negative check-point regulators of the cell cycle. We therefore aimed to evaluate p21 and p27 expression in oral squamous cell carcinomas (OSSC) to determine the value as a prognostic marker. One hundred and ninety-two patients with histologically proven, surgically treated squamous cell carcinoma of the oral cavity were eligible for the study and investigated for the expression of p21 and p27 by means of tissue microarrays (TMAs). Immunohistochemical screening under identical condition were carried out with antibodies against p21 and p27. Correlations between clinical features and the expression of the respective antibodies were evaluated statistically by Kaplan-Meier curves, log-rank and $\chi^{2}$ tests. The expression of p21 correlated significantly with an increased prognosis in the log-rang test $(\mathrm{p}=0.01)$. No significant correlation was found between the expression of $\mathrm{p} 27$ and the overall survival rate. In multivariate Cox analysis, p27 was indicated as independent predictor of survival prognosis in the subgroup of nodal positive carcinomas, p27 positive tumours showed a significantly better survival prognosis $(\mathrm{p}=0.03) \cdot \mathrm{p} 21$ and $\mathrm{p} 27$ in carcinoma of oral cavity seem to be predictive parameter in regulation and prognosis of squamous cell carcinomas. A p21 negative subgroup of OSCC may benefit from additional radio or radiochemotherapy.
\end{abstract}

\section{Introduction}

Carcinomas of the head and neck represent the sixth most frequent cancer worldwide, and at least $85 \%$ of them are

Correspondence to: Dr Thomas Fillies, Department of CranioMaxillofacial Surgery, University of Münster, Waldeyerstrasse 30, D-48129 Münster, Germany

E-mail: fillies@uni-muenster.de

Key words: p21, p27, oral squamous cell carcinoma squamous cell carcinomas (1). An improved understanding of the biology of the squamous cell carcinoma (SCC) of the oral cavity is urgently required because the prediction of the individual patient outcome is largely unpredictable.

Genes and the respective gene products are an important clue in order to understand the biology and the associated prognosis. Cell-cycle control is superordinated by cyclins and cyclin-depent kinases (cdk). The activity of these enzymes is restricted by the inhibitory action of the CipKip family (2). Members of the CipKip family, p21 $1^{\text {WaflCip } 1}$ and $27^{\text {Kip } 1}$ block the activity of CDK2 in the cyclin E/A-CDK2 complexes (2-4). Cdkis influence the critical transition from the G1 phase of the cell cycle to the $\mathrm{S}$ phase. P21 is regulated in two different ways through a p53-dependent and a p53-independent pathway. Cellular DNA damage leads via p53-activation to an up-regulation of p21 to cause cell-cycle arrest in the G1 phase with the cellular possibility for DNA-repair or the induction of apoptosis (5). In addition, p21 can be regulated independent of p53 by cellular growth factors (6).

$\mathrm{p} 27$ is a structural homolog to $\mathrm{p} 21$. The expression of p27 is increased by growth inhibition signals and leads to cell-cycle arrest (7), on the other hand it is decreased by growth stimulating signals leading to cell cycle progression (8). At present, the prognostic value of p21 and p27 in HNSCC is discussed controversially.

The aim of this study was to evaluate the aberrant expression of the cell cycle associated proteins p21 and p27 in oral squamous cell carcinomas in order to determine their value as prognostic markers in a large series of HNSCC.

\section{Materials and methods}

Patients and tumour material. Formalin-fixed, paraffinembedded archival tumour tissue from 192 patients with primary SCC of the oral cavity was obtained from the archives of the Institute of Pathology, University of Münster, Germany. Clinicopathological details are shown in Table I.

Patients with histologically proven squamous cell carcinoma of the oral cavity treated surgically were eligible for the study. Surgical treatment included radical tumour resection of the whole tumour with a free histopathological proven margin of at least $4 \mathrm{~mm}$ from the tumour borders. Selective neck dissection of levels I, II, III and V was performed in 
Table I. Clinicopathological data.

Age (mean years)

59 (range 31-90)

$\operatorname{Sex}(M / F)$

Follow-up (mean months)

59.5 (range 4-181)

T stage (tumours)

$\mathrm{T} 1$

95

$\mathrm{T} 2$

T3-T4

$\mathrm{N}$ stage

Lymph node negative carcinoma

Lymph node positive carcinoma

Grading

Well-differentiated tumours (G1)

Moderately differentiated tumours $(\mathrm{G} 2)$

Poorly differentiated tumours (G3)

Recurrent disease

OSCC developed a recurrent tumour

Disease-free in follow-up

case of suspect results in preoperative tumour staging by computer-tomography or sonography examination or in case of tumour size over $2 \mathrm{~cm}$, bilateral selective neck dissection was performed when the tumour spread the midline [according to the recommendation of Robins et al (9)]. Radiotherapy was performed when lymph node metastases were detected histologically. The series comprised 192 patients (154 men and 38 women) with a median age of 59.5 years (range 31-90). All tumours were classified post-surgically according to the TNM system (10). Patients were clinically evaluated in our routine follow-up for 3-5 years.

Method. Tumour specimens of 192 patients were investigated for the expression of p21 and p27. We used tissue microarrays (TMAs) and immunhistochemistry in order to investigate all tumour specimens under identical conditions.

For TMA construction, two punch biopsies with a diameter of $0.6 \mathrm{~mm}$ from each donor block were taken and transferred into the new acceptor block. TMA construction was performed by using a special tissue microarray instrument (Beecher Instruments, New Jersey, USA), according to standard protocols $(11,12)$.

Immunohistochemistry was performed on $4-\mu$ m-thick slides. After deparaffinization and rehydration, endogenous peroxidase activity was blocked for $30 \mathrm{~min}$ in methanol containing $0.3 \%$ hydrogen peroxide. After antigen retrieval (microwave $10 \mathrm{~min}$, citrate buffer), a cooling-off period of 20 min preceded the incubation of the primary antibody. The primary antibodies for this study were used in the following dilutions: anti-p21, Pharmingen, dilution 1:500; anti-p27, Pharmingen, dilution 1:1000.

The antibodies were detected by a standard avidin-biotin complex method with a biotinylated rabbit anti-mouse antibody

(Dako) and an avidin-biotin complex (Dako). The staining was developed with diaminobenzidine. Before the slides were mounted, all sections were counterstained for $45 \mathrm{sec}$ with hematoxylin and dehydrated in alcohol and xylene. Appropriate negative controls (obtained by omission of the primary antibody) and positive controls were used throughout.

The expression of p21 and p27 was determined independently by two pathologists. Both pathologists determined the percentage of staining intensity in each core. The mean percentage value of two cores representing one tumour was used for further evaluation.

The immunostaining was classified for each antibody. Loss of expression was determined for $<1 \%$ and positive expression when the cells showed $\geq 1 \%$ positively stained nuclei.

Statistical analysis. All calculations were performed using the statistical computer program SPSS 12.01 (USA). The expression of the categorical variants of p21 and p27 and clinicopathological parameters was examined by the $\chi^{2}$ test. Cumulative survival was analyzed using the method of KaplanMeier (13), a comparison between groups was done with the log-rank test, the multivariate survival analysis was performed with Cox regression (14), a p-value $<0.05$ was considered to be significant.

The duration of survival was measured from the date of surgery, to the date of histologically proven recurrent or metastatic carcinoma or to the time of death or at 60 -month last follow-up. Patients lost to follow-up were censored at the date of the last examination. The statistical significance was set at $\mathrm{p}<0.05$.

\section{Results}

p21. One hundred and eighty-two tumour samples could be evaluated for the expression of p21 and p27. Ten tumour samples were lost from the slides during the immunohistochemical staining procedures.

Immunohistochemical examination showed that p21 and p27 reactivity was confined to the nuclei of neoplastic cells (Fig. 1). The expression levels did not differ significantly for the vast majority of the cases between the two tumour cores repre-senting one case, indicating a rather homogeneous expression. The difference between the evaluations of expression from both pathologists was within the range of $10 \%$.

Expression levels of p21 (p21 $\geq 1 \%$ ) were found in 52/182 $(29 \%)$ of oral SCCs. No p21 expression (p21<1\%) was found in $130 / 182$ oral SCCs $(71 \%)$.

Kaplan-Meier analysis of p21 expression showed a significantly decreased overall survival $(\mathrm{p}=0.01)$ of carcinomas with loss of p21 expression (p21<1\%) (Fig. 2). The expression levels of p21 showed no association with the disease-free survival. p21 expression was inversely correlated with tumour size in the $\chi^{2}$ test $(\mathrm{p}<0.01)$.

p27. Detectable levels of p27 (p27 $\geq 1 \%$ ) were found in 38/ 189 of the oral SCCs $(20 \%)$. No expression level of p27 (p27 $<1 \%$ ) was found in $151 / 189$ oral SCCs $(80 \%)$. No statistical association was found between p27 expression and the overall 


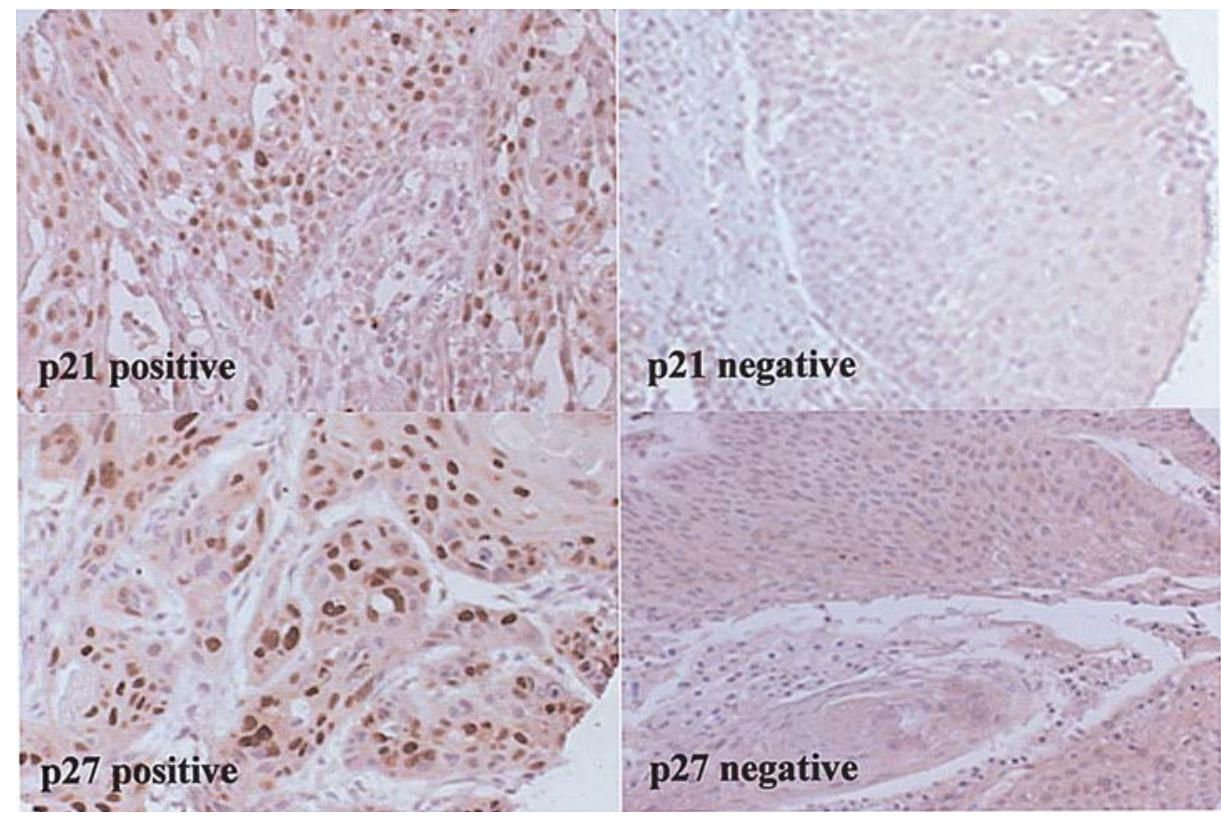

Figure 1. Immunohistochemical staining of OSCC with p21 and p27 (left, positive expression; right, no expression); magnification, x20.

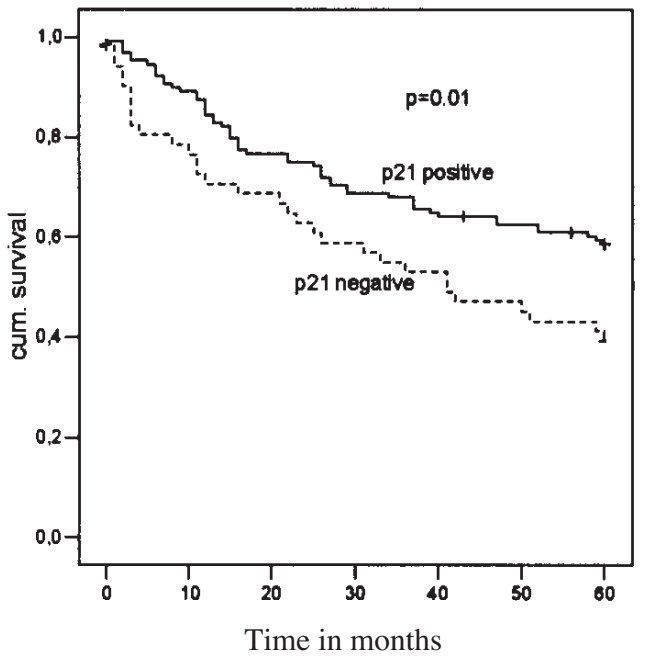

Figure 2. Kaplan-Meier survival curve in oral SCC with p21 dependent expression.

survival of OSCC's. In the nodal positive subgroup of tumours, the Kaplan-Meier analysis showed a significantly decreased survival $(\mathrm{p}=0.03)$ of carcinomas with lack of $\mathrm{p} 27$ expression (p27 <1\%) (Fig. 3). No correlation could be elaborated between p27 expression and the disease-free survival.

No statistically significant differences were found between tumour size, tumour differentiation, lymph node status and the expression of $\mathrm{p} 27$ in the $\chi^{2}$ test.

In combination of p21 and p27 we found a significant decreased survival for $\mathrm{p} 21$ negative OSCC in the $\mathrm{p} 27$ negative subgroup $(\mathrm{p}<0.01)$ (Fig. 4). In the opposite combination of p21 negative subgroup we found no significant association between the expression patterns.

In multivariate Cox analysis we compared the survival according to the clinical prognostic factors of tumour size and nodal status with p21, p27. The nodal status, tumour size

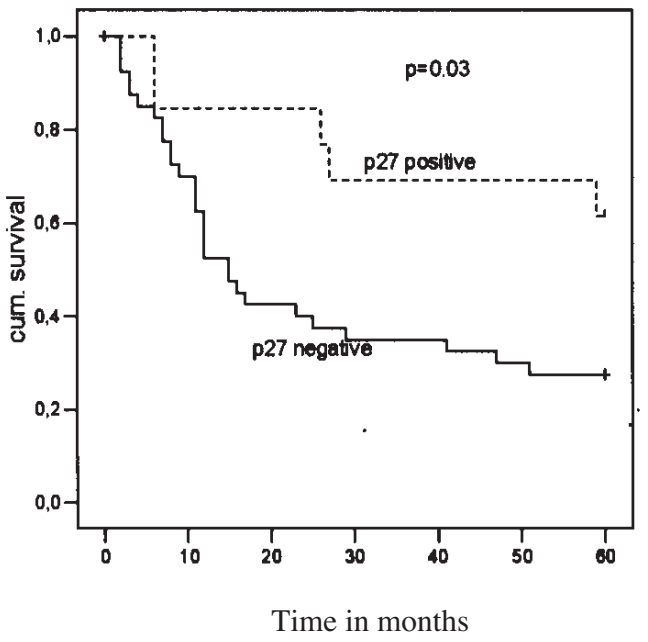

Figure 3. Kaplan-Meier survival curve in node-positive oral SCC with p27 dependent expression.

and p27 were indicated by Cox regression as independent predictors of survival prognosis (Table II).

\section{Discussion}

The individual prediction of local and metastatic tumour control by surgery is an unresolved problem. Currently, only the tumour stage, the localisation of the primary tumour and the presence of nodal or distant metastasis affects strategy of therapy in head and neck squamous cell carcinoma. In the large group of HNSCC, not only tumours of different stage but also tumours of same stage and localization show a different biological behaviour and clinical outcome.

Histopathological properties of oral carcinoma may therefore be of valuable help in defining biological parameters which might explain these clinical discrepancies. Reliable 


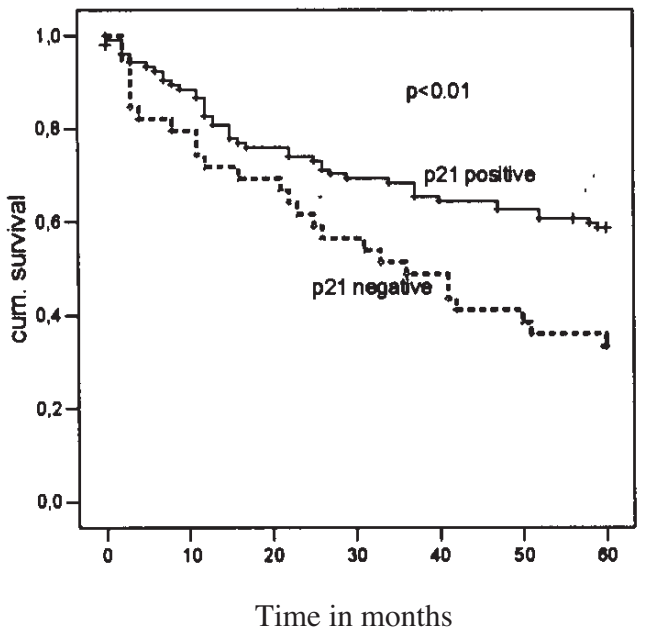

Figure 4. Kaplan-Meier survival curve of p27-negative OSCC with p21 dependent expression.

Table II. Multivariate Cox analysis.

\begin{tabular}{lccc}
\hline Prognosticator & P-value $^{\mathrm{a}}$ & $\mathrm{RR}^{\mathrm{b}}$ & Confidance interval $^{\mathrm{c}}$ \\
\hline Tumour size & 0.02 & 1.6 & $1.1-2.3$ \\
Nodal status & 0.00 & 1.8 & $1.3-2.5$ \\
Grading & 0.44 & 0.8 & $0.5-1.3$ \\
p21 & 0.43 & 0.8 & $0.5-1.4$ \\
p27 & 0.04 & 0.5 & $0.2-0.9$ \\
\hline
\end{tabular}

${ }^{a}$ The partially non-parametric regression model of Cox (1972) was used to evaluate the predictive power of various combinations of prognosticators in a multivariate manner. ${ }^{\mathrm{b}} \mathrm{RR}$, relative risk. ${ }^{\mathrm{c} 95 \%}$ confidence interval.

predictors of treatment outcome are needed to optimize therapy strategies.

Cell-cycle regulators and the involved genes represent an important field for the investigation of possible prognostic factors and indicators for advanced therapeutic strategies in HNSCC.

In our investigation we were able to show a decreased clinical outcome for p21 negative squamous cell carcinomas of the oral cavity $(\mathrm{p}=0.01)$. The existing literature presents controversial results of clinical outcome dependent on the value of p21 expression in HNSCC (15-18). Similar results to our study are shown by Xie et al (18). An association was demonstrated between $\mathrm{p} 21$ expression and increased survival in carcinoma of the tongue, as well as an association between p21 expression level and the tumour stage (18). From a tumour biological point of view, these findings seem logical since the loss of inhibition of Cdk influences the critical transition from the G1 phase of the cell cycle to the $\mathrm{S}$ phase. Some discrepancies concerning other studies might be flawed by the heterogeneous tumour series, since laryngeal and oral SCCs were mixed. However, taking into account all results for oral carcinomas a trend is obvious supporting an association between p21 expression and the clinical outcome of HNSCCs. Similar results have been reported in other solid tumour entities (17-19). Biological hints supporting this important position of p21 already exist. Kapranos et al showed that p21 plays a key role in the successful response to chemotherapy. This association could be of interest for tumour therapy in OSCC (17). For colorectal cancer an enhanced apoptotic effect of p21 negative cells of chemotherapeutic agents and $\gamma$-irradiation has been shown as compared to p21 colorectal cancer cells (20). A xenograft model indicated that $\mathrm{p} 21$-deficient tumours were more sensitive to radiation (21). Another approach is seen with antisense-oligonucleotides to p21 which also enhanced $\gamma$ irradiation-induced apoptosis and cytotoxicity as shown in radio resistant human glioma cells (22). p21 seems a possible candidate to indicate radiotherapy or radiochemotherapy of the p21 negative tumour subgroup independent of the lymph node status to improve the clinical outcome.

For p27 we failed to demonstrate a general correlation between loss of p27 expression and overall survival. However, we were able to demonstrate in a subgroup of nodal positive oral SCC a decreased survival rate in cases with loss p27 $(\mathrm{p}=0.03)$. In the $\mathrm{p} 27$ negative subgroup we found a worse survival rate in combination with $\mathrm{p} 21$ negative tumours. The opposite combination of the p21 negative subgroup with $\mathrm{p} 27$ shows no significance. In multivariate Cox regression the nodal status, tumour size and p27 were indicated as independent predictors of survival prognosis.

Down-regulation of $\mathrm{p} 27$ protein was frequently found in early invasive OSCC limited to the mucosa. Several groups observed a reduced expression of p27 in severe epithelial dysplasias and decreased p27 expression with progression of OSCC. Kudo et al (23) demonstrated a reduced expression of p27 frequently found in OSCC and was well correlated with early invasion and poor prognosis of OSCC $(24,25)$. Experimentally, it has been shown that antisense-oligonucleotidemediated down-regulation of p27 in EMT-6 mammary tumour cell spheroids reduced intercellular adhesion and increased cell proliferation.

Several studies of OSCC have shown a significant association between the loss of p27 expression and decreased overall survival rate (26-28). The double negative subgroup for p27 and p21 in our investigation seems to demonstrate an additional subgroup where an advanced therapeutic strategy may benefit the clinical outcome. The results of the multivariate analysis are however unclear.

In summary, we were able to show that the expression of p21 is associated with an improved prognosis in patients with OSCC, irrespective of the primary lymph node status. The postulation of $\mathrm{p} 21$ in a clinical setting as a reliable prognostic marker and indicator for radio or radio-chemotherapy in p21 negative OSCC will require more additional clinical and molecular research efforts.

\section{References}

1. Hoffman HT, Karnell LH, Funk GF, Robinson RA and Menck HR: The National Cancer Data Base report on cancer of the head and neck. Arch Otolaryngol Head Neck Surg 124: 951-962, 1998. 
2. Sherr CJ and Roberts JM: CDK inhibitors: positive and negative regulators of G1-phase progression. Genes Dev 13: 1501-1512, 1999.

3. Morgan DO: Cyclin-dependent kinases: engines, clocks and microprocessors. Annu Rev Cell Dev Biol 13: 261-291, 1997.

4. Wuarin J and Nurse P: Regulating S phase: CDKs, licensing and proteolysis. Cell 85: 785-787, 1996.

5. Hartwell LH and Kastan MB: Cell cycle control and cancer. Science 266: 1821-1828, 1994.

6. Cordon-Cardo C: Mutations of cell cycle regulators. Biological and clinical implications for human neoplasia. Am J Pathol 147: 545-560, 1995.

7. Polyak K, Kato JY, Solomon MJ, Sherr CJ, Massague J, Roberts JM and Koff A: p27Kip1, a cyclin-Cdk inhibitor, links transforming growth factor-beta and contact inhibition to cell cycle arrest. Genes Dev 8: 9-22, 1994.

8. Coats S, Flanagan WM, Nourse J and Roberts JM: Requirement of p27Kip1 for restriction point control of the fibroblast cell cycle. Science 272: 877-880, 1996.

9. Robbins KT, Clayman G, Levine PA, Medina J, Sessions R, Shaha A, Som P and Wolf GT: American Head and Neck Society; American Academy of Otolaryngology-Head and Neck Surgery: Neck dissection classification update: revisions proposed by the American Head and Neck Society and the American Academy of Otolaryngology-Head and Neck Surgery. Arch Otolaryngol Head Neck Surg 128: 751-758, 2002.

10. Wittekind C, Klimpfinger M and Sobin LH: TNM - Atlas. 5. Aufl., Springer, Berlin, 2005.

11. Kononen J, Bubendorf L, Kallioniemi A, et al: Tissue microarrays for high-throughput molecular profiling of tumour specimens. Nat Med 4: 844-847, 1998.

12. Fillies T, Werkmeister R, Packeisen J, Brandt B, Morin P, Weingart D, Joos U and Buerger H: Cytokeratin 8/18 expression indicates a poor prognosis in squamous cell carcinomas of the oral cavity. BMC Cancer 13: 10, 2006.

13. Kaplan EL and Meier P: Non-parametric estimation from incomplete observations. J Am Stat Ass 53: 457-481, 1958.

14. Cox DR: Regression models and life-tables. J R Stat Soc (B) 34: 187-202, 1972.

15. Miracca EC, Kowalski LP and Nagai MA: High prevalence of p16 genetic alterations in head and neck tumours. Br J Cancer 81: 677-683, 1999.

16. Li W, Thompson CH, O'Brien CJ, McNeil EB, Scolyer RA, Cossart YE, Veness MJ, Walker DM, Morgan GJ and Rose BR: Human papillomavirus positivity predicts favourable outcome for.squamous carcinoma of the tonsil. Int J Cancer 106: 553-558, 2003.
17. Kapranos N, Stathopoulos GP, Manolopoulos L, et al: p53, p21 and p27 protein expression in head and neck cancer and their prognostic value. Anticancer Res 21: 521-528, 2001.

18. Xie X, Clausen OP and Boysen M: Prognostic significance of p21WAF1/CIP1 expression in tongue squamous cell carcinomas. Arch Otolaryngol Head Neck Surg 128: 897-902, 2002.

19. Winters ZE, Leek RD, Bradburn MJ, Norbury CJ and Harris AL: Cytoplasmic p21Waf1/Cip1 expression is correlated with HER-2/ neu in breast cancer and is an independent predictor of prognosis. Breast Cancer Res 5: 242-249, 2003.

20. Waldman T, Lengauer C, Kinzler KW and Vogelstein B: Uncoupling of $\mathrm{S}$ phase and mitosis induced by anticancer agents in cells lacking p21. Nature 381: 713-716, 1996.

21. Wouters BG, Giaccia AJ, Denko NC and Brown JM: Loss of p21Waf1/Cip1 sensitizes tumors to radiation by an apoptosisindependent mechanism. Cancer Res. 57: 4703-4706, 1997.

22. Kokunai T, Urui S, Tomita $\mathrm{H}$ and Tamaki N: Overcoming of radioresistance in human gliomas by 21 WAF1/CIP1 antisense oligonucleotide. J Neurooncol 51: 111-119, 2001

23. Kudo Y, Kitajima S, Sato S, Miyauchi M, Ogawa I and Takata T: High expression of S-phase kinase-interacting protein 2, human F-box protein, correlates with poor prognosis in oral squamous cell carcinomas. Cancer Res 61: 7044-7047, 2001.

24. Kudo Y, Takata T, Yasui W, et al: Reduced expression of cyclindependent kinase inhibitor p27Kip1 is an indicator of malignant behavior of oral squamous cell carcinomas. Cancer 83: 2447-2455, 1998.

25. Kudo Y, Takata T, Ogawa I, et al: Reduced expression of p27(Kip1) correlates with an early stage of cancer invasion in oral squamous cell carcinoma. Cancer Lett 151: 217-222, 2000.

26. Shintani S, Li C, Mihara M, Hino S, Nakashiro K and Hamakawa H: Skp2 and Jab1 expression are associated with inverse expression of $\mathrm{p} 27 \mathrm{KIP} 1$ and poor prognosis in oral squamous cell carcinomas. Oncology 65: 355-362, 2003.

27. Mineta H, Miura K, Suzuki I, et al: Low p27 expression correlates with poor prognosis for patients with oral tongue squamous cell carcinoma. Cancer 85: 1011-1017, 1999.

28. Venkatesan TK, Kuropkat C, Caldarelli DD, et al: Prognostic significance of p27 expression in carcinoma of the oral cavity and oropharynx. Laryngoscope 109: 1329-1333, 1999. 